\title{
Mutant p53 controls tumor metabolism and metastasis by regulating PGC-1a
}

\author{
Subhasree Basu, ${ }^{1}$ Keerthana Gnanapradeepan, ${ }^{1,2,7}$ Thibaut Barnoud ${ }^{1,7}$ Che-Pei Kung, ${ }^{1,5,7}$ \\ Michele Tavecchio, ${ }^{3,6}$ Jeremy Scott, ${ }^{1}$ Andrea Watters, ${ }^{3}$ Qing Chen, ${ }^{3}$ Andrew V. Kossenkov, ${ }^{4}$ \\ and Maureen E. Murphy ${ }^{1}$

\begin{abstract}
${ }^{1}$ Program in Molecular and Cellular Oncogenesis, The Wistar Institute, Philadelphia, Pennsylvania 19104, USA; ${ }^{2}$ Graduate Group in Biochemistry and Biophysics, The University of Pennsylvania Perelman School of Medicine, Philadelphia, Pennsylvania 19104; ${ }^{3}$ Program in Tumor Microenvironment and Metastasis, The Wistar Institute, Philadelphia, Pennsylvania 19104, USA; ${ }^{4}$ Program in Center for Systems and Computational Biology, The Wistar Institute, Philadelphia, Pennsylvania 19104, USA
\end{abstract}

\begin{abstract}
Mutant forms of p53 protein often possess protumorigenic functions, conferring increased survival and migration to tumor cells via their "gain-of-function" activity. Whether and how a common polymorphism in TP53 at amino acid 72 (Pro72Arg; referred to here as P72 and R72) impacts this gain of function has not been determined. We show that mutant 53 enhances migration and metastasis of tumors through the ability to bind and regulate PGC-1a and that this regulation is markedly impacted by the codon 72 polymorphism. Tumor cells with the R72 variant of mutant p53 show increased PGC-1 a function along with greatly increased mitochondrial function and metastatic capability. Breast cancers containing mutant $\mathrm{p} 53$ and the $\mathrm{R} 72$ variant show poorer prognosis compared with $\mathrm{P} 72$. The combined results reveal PGC-1 $\alpha$ as a novel "gain-of-function" partner of mutant p53 and indicate that the codon 72 polymorphism influences the impact of mutant p53 on metabolism and metastasis.
\end{abstract}

[Keywords: mutant p53; codon 72; metastasis; oxidative phosphorylation; PGC-1a]

Supplemental material is available for this article.

Received October 30, 2017; revised version accepted January 22, 2018.

Many years ago, it was reported that introduction of a tumor-derived mutant form of p53 into a p53-null tumor cell line rendered the tumor cells markedly more lethal in recipient mice (Wolf et al. 1984). Subsequent studies confirmed that introduction of any of a series of tumorderived missense mutant forms of p53 led to marked increases in the tumorigenic properties of p53-null cancer cell lines (Dittmer et al. 1993). In recent years, significant efforts from multiple groups have aimed to understand how mutant forms of p53 confer enhanced tumorigenic properties. The combined research supports the following two premises: The first is that tumor cells with mutant forms of p53 are addicted to this protein. For example, silencing mutant p53 in tumor cell lines inhibits their proliferation (Olive et al. 2004). Similarly, treatment of tumor cells with HSP90 inhibitors leads to degradation of mutant p53 and consequent cell death (Alexandrova et al. 2015). The second premise is that tumors appear to be addicted to mutant p53 because these mutant proteins possess protumorigenic or "gain-of-function" activities. Mutant p53 "gain of function" is best exemplified in

Present addresses: ${ }^{5}$ Washington University, St Louis, MO 63110, USA; ${ }^{6}$ Mitochondrial Medicine Laboratory, Lund University, Lund SE 221 00, Sweden.

${ }^{7}$ These authors contributed equally to this work.

Corresponding author: mmurphy@wistar.org

Article published online ahead of print. Article and publication date are online at http://www.genesdev.org/cgi/doi/10.1101/gad.309062.117. mouse models, where mice that express tumor-derived mutant forms of p53 have an increased tumor spectrum and significantly more metastatic tumors compared with p53-null mice (Lang et al. 2004; Olive et al. 2004).

There are some known mechanisms by which mutant p53 confers protumorigenic function. The first is through the ability of mutant p53 to bind and inhibit the p53 family members p63 and p73, which themselves possess some tumor-suppressive function (Di Como et al. 1999; Gaiddon et al. 2001; Lang et al. 2004; Olive et al. 2004). For example, the ability of mutant p53 to bind and inhibit p63 leads to the decreased ability of p63 to regulate the recycling of integrin receptors (Muller et al. 2009) and receptor tyrosine kinases such as MET (Muller et al. 2013). This leads to increased migration and metastasis in tumor cells with mutant p53. Similarly, inhibition of p63 by mutant p53 also leads to the decreased ability of p63 to transactivate the p63 target genes Sharp-1 and Cyclin G2, both of which inhibit metastasis (Adorno et al. 2009). Inactivation of $\mathrm{p} 73$ by mutant $\mathrm{p} 53$ causes increased PDGF receptor $\beta$ (PDGFR $\beta$ ) signaling and metastasis (Weissmueller et al. 2014). Other studies have revealed

(C) 2018 Basu et al. This article is distributed exclusively by Cold Spring Harbor Laboratory Press for the first six months after the full-issue publication date (see http://genesdev.cshlp.org/site/misc/terms.xhtml). After six months, it is available under a Creative Commons License (Attribution-NonCommercial 4.0 International), as described at http://creativecommons.org/licenses/by-nc/4.0/. 
that mutant forms of p53 can bind to and enhance the stability/activity of certain protumorigenic transcription factors, including ETS2 (Zhu et al. 2015), NF-kB (Cooks et al. 2013), and SREBP1/2 (Freed-Pastor et al. 2012). Mutant p53 also can influence the global chromatin remodeler SWI-SNF (Pfister et al. 2015). Finally, mutant forms of p53 can increase glucose consumption by altering GLUT-1 trafficking to the plasma membrane (Zhang et al. 2013). These and other activities of mutant p53 have been demonstrated to contribute to the enhanced tumorigenic properties of tumor cells containing missense mutations in $\mathrm{p} 53$.

A common single-nucleotide polymorphism (SNP) exists in TP53 at codon 72 that alters the function of wildtype p53 (Basu and Murphy 2016). This SNP may also be an intragenic modifier of the "gain-of-function" effect of mutant p53, with the R72 variant showing an enhanced ability to bind and inactivate p73 relative to the proline 72 variant (P72) (Marin et al. 2000). In individuals heterozygous for this variation $(\mathrm{P} / \mathrm{R})$, there is some evidence for preferential retention of the mutant $\mathrm{R} 72$ allele, with loss of the P72 allele in tumors (Marin et al. 2000). It has been hypothesized that tumors with the R72 form of mutant p53 (R72-mut-p53) might show a worse prognosis, but this premise was never firmly validated. Furthermore, the impact of the codon 72 polymorphism on other "gain-of-function" activities of mutant p53 has not been assessed.

The Warburg effect refers to the observation made in the 1920s by Otto Warburg that tumor cells preferentially use aerobic glycolysis for ATP generation instead of oxidative phosphorylation. Wild-type p53 is known to repress the Warburg effect in part through its ability to transactivate target genes that are required for oxidative phosphorylation such as SCO2 (Matoba et al. 2006) as well as genes such as $R R A D$ that encode an inhibitor of glycolysis (Zhang et al. 2014). Mutant p53 can enhance "Warburg" metabolism by increasing glucose uptake in a manner that requires GLUT1 and RhoA/ROCK (Zhang et al. 2013). It might be reasonable to expect that in order to fully favor aerobic glycolysis in tumor cells, mutant p53 might not only enhance glycolysis but also actively inhibit oxidative phosphorylation. We found this to be the case. In this study, we used an unbiased approach to show that several different tumor-derived mutant forms of p53 bind and inhibit PGC-1 $\alpha$, a master regulator of mitochondrial biogenesis and oxidative phosphorylation. Furthermore, we show that the codon 72 variation markedly impacts this inhibition, with the P72 variant of mutant p53 (P72mut-p53) being markedly more effective at binding and inhibiting PGC-1 a function. Conversely, we found that cells containing the R72 variant of mutant p53 (R72-mut-p53) show increased PGC-1a function and oxidative phosphorylation as well as increased metastatic ability. These results demonstrate a new function of mutant p53 in the control of the Warburg effect in cancer cells. Our results also reveal a new effect of the codon 72 variant of p53 on cancer metabolism and metastasis. Both of these findings have an impact for our understanding of cancer progression and prognosis.

\section{Results}

Increased migration and invasion in tumor cells with R72-mut-p53

Mutant p53 drives cancer cell migration and invasion (Adorno et al. 2009; Muller et al. 2009, 2013; Wang et al. 2009). However, the influence of the codon 72 polymorphism on the ability of mutant p53 to confer these activities has not been fully investigated. To address this issue, we created matched cell lines in three different p53-null cancer cell lines (H1299 lung adenocarcinoma, Saos2 osteosarcoma, and PC3 prostate carcinoma) containing three different tumor-derived mutant forms of p53: two "hot spot" mutants (R175H and R273H) and one Li Fraumeni mutant (A138V), all in cis with either P72 or R72. For simplicity, these are referred to here as P72-mut-p53 and R72-mut-p53. Alternatively, where relevant, the specific mutation is denoted (e.g., P72-R175H and $\mathrm{R} 72-175 \mathrm{H})$. For all studies, we collected data from a minimum of two independent clones for each mutant cell line; these clones were selected to express similar levels of mutant p53, and all cells stained positively for the presence of mutant p53 (Fig. 1A,C; Supplemental Fig. 1A). These cell lines, along with the vector transfected control parental lines, were subjected to scratch/ wound healing assays to examine cell migration. The results of these scratch/wound healing assays indicated that R72-mut-p53 showed significantly increased migration compared with the P72-mut-p53; this was true for all clones, all three backgrounds, and all three tumor-derived mutations in p53. Overall, both the velocity of wound healing and the percentage of the wound closed at each time point were increased for all forms of R72mut-p53 in all cell backgrounds (Fig. 1B,D; Supplemental Fig. 1B,C).

We next examined Boyden chamber assays for cell invasion potential. These analyses revealed that for all three cell backgrounds and all three p53 mutants, cells containing R72-mut-p53 showed increased invasion through Matrigel (Fig. 1E,F,H; Supplemental Fig. 1D-G). When we silenced mutant p53 using a short hairpin in the $\mathrm{H} 1299$ lines containing the R175H and R273H mutants, this effectively equalized the invasive ability of all of the clones (Fig. 1G,H). We next analyzed the proliferation rates of these cell lines as controls for these experiments. This analysis revealed that cells containing mutant p53 showed enhanced cell proliferation compared with the p53-null vector transfected parental cells; however, we found no evidence for an impact of the codon 72 polymorphism of mutant p53 on cell proliferation (Supplemental Fig. $1 \mathrm{H}, \mathrm{I})$. To further support these findings, we analyzed two colon cancer cell lines containing the endogenous p53 mutation R273H: HT-29 (P72-R273H) and SW-620 (R72$\mathrm{R} 273 \mathrm{H})$. We found that SW-620 (R72-mut-p53) showed increased invasiveness compared with HT-29 (P72-mutp53) (Fig. 1I,J). Silencing mutant p53 equalized their invasiveness (Fig. 1K). Our combined data support the premise that tumor cell lines containing the R72-mut-p53 protein show enhanced migration and invasion compared with lines containing P72-mut-p53. 


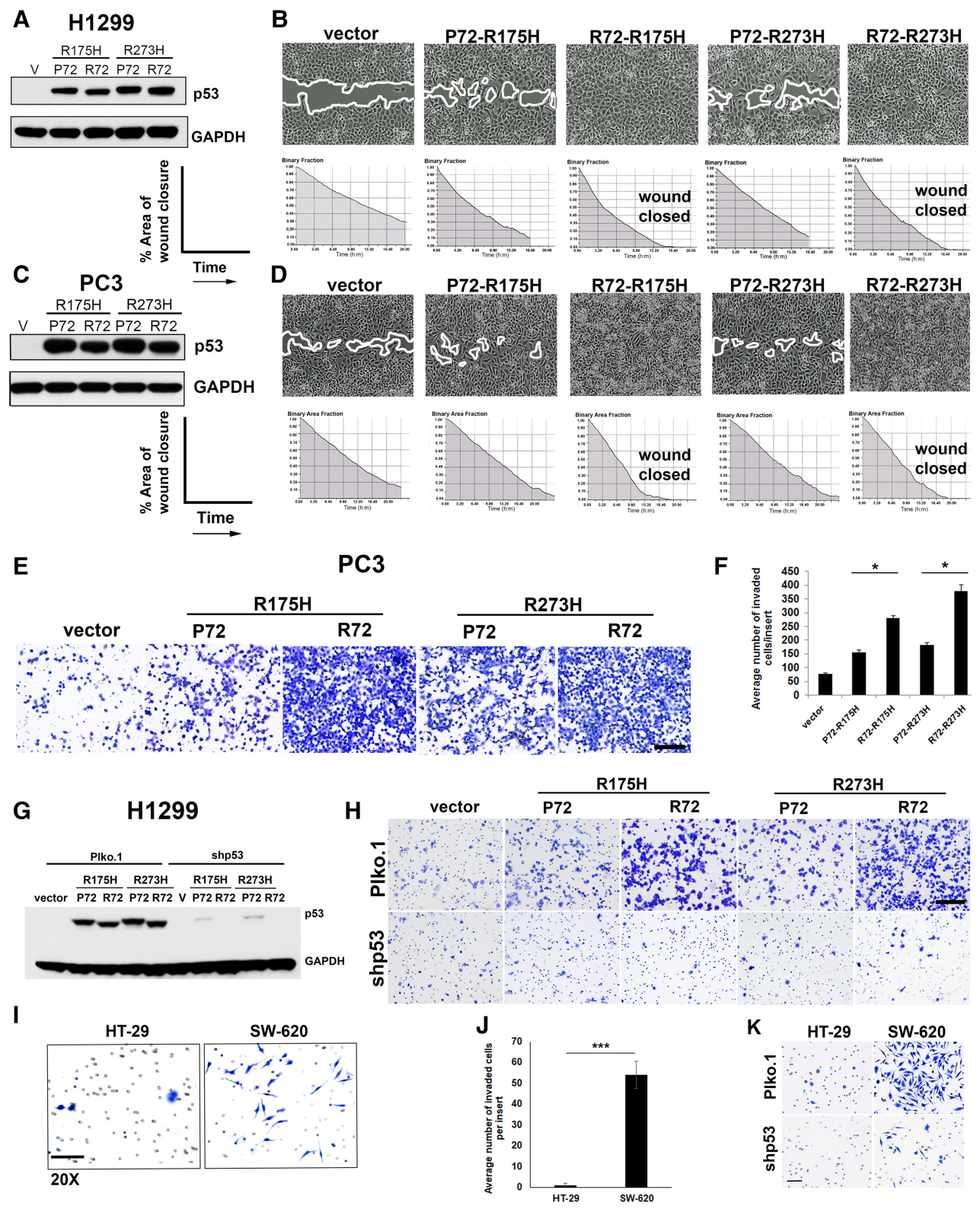

Figure 1. Tumor cells containing R72-mut-p53 show increased migration and invasion compared with P72-mut-p53. (A) Western blot analysis of whole-cell lysates to detect mutant p53 levels from H1299 cells expressing either the proline (P72) or arginine (R72) variant for the hot spot mutations $\mathrm{R} 175 \mathrm{H}$ and $\mathrm{R} 273 \mathrm{H}$. Note that the $\mathrm{P} 72$ variant routinely runs at a higher molecular weight than the R72 variant. GAPDH was used as a loading control. (B) Scratch/wound healing assay in H1299 cells expressing the vector (control) and either P72 or $\mathrm{R} 72$ variants for both $\mathrm{R} 175 \mathrm{H}$ and $\mathrm{R} 273 \mathrm{H}$. The wound healing assay was performed on each cell type for $24 \mathrm{~h}$, and time-lapse phase-contrast microscopy was used to examine the percentage of wound closure ( $Y$-axis) with progressive time ( $X$-axis). ( $C)$ Western blot analysis in PC3 cells to test mutant p53 levels in PC3 cells expressing either the P72 or R72 variants for both R175H and R273H mutations. GAPDH was used as a loading control. $(D)$ Scratch/wound healing assay in PC3 cells expressing the vector control or either the P72 or R72 variants of the $\mathrm{R} 175 \mathrm{H}$ and $\mathrm{R} 273 \mathrm{H}$ mutants of $\mathrm{p} 53$. Phase-contrast microscopy was performed for $24 \mathrm{~h}$ to monitor the percentage of wound closure. $(E)$ Representative images of Boyden chamber inserts stained with crystal violet showing invaded PC3 cells (vector and either the P72 or the $\mathrm{R} 72$ variant of the $\mathrm{R} 175 \mathrm{H}$ and $\mathrm{R} 273 \mathrm{H}$ mutations). Bar, $100 \mu \mathrm{m}$. (F) Quantification of the average number of invaded PC3 cells in the Boyden chamber assay. Results are representative of three independent experiments and two independent clones of each genotype. Error bars represent standard deviation (SD). $\left.\left.\right|^{*}\right) P<0.05$. $(G)$ Western blot analysis in H1299 cells infected with either the control PLKO.1 or the short hairpin to p53. Lysates were analyzed to detect levels of mutant p53. GAPDH was used as a loading control. $(H)$ Representative images of H1299 cells stained with crystal violet that have invaded through the Boyden chamber inserts. Cells (vector and either the P72 or the R72 variant of the $\mathrm{R} 175 \mathrm{H}$ and $\mathrm{R} 273 \mathrm{H}$ mutations) were infected with either the control short hairpin PLKO.1 or the p53 short hairpin (shp53). Bar, $100 \mu \mathrm{m}$. All results are representative of three independent experiments, each analyzing two independent clones. $(I)$ Representative crystal violet-stained images of HT-29 and SW-620 cells that have invaded through Boyden chamber inserts. Images are shown at high $(20 \times)$ magnification. Bar, $100 \mu \mathrm{m}$. (J) Quantification of average number of HT-29 and SW-620 cells that have invaded through the inserts. Results are representative of three technical replicates. Error bars represent standard error of the mean $(\mathrm{SEM}) .\left(^{* * *}\right) P<0.001$. $(K)$ Representative crystal violet-stained images of HT-29 and SW-620 cells that were infected with PLKO.1 (control) or p53 short hairpin (shp53) and that have invaded through Boyden chamber inserts. Bar, $100 \mu \mathrm{m}$. 
Enhanced metastasis in tumor cell lines containing R72-mut-p53

We next sought to test the impact of the codon 72 polymorphism of mutant p53 on lung metastasis using H1299 cells containing the P72 and R72 versions of the $\mathrm{R} 175 \mathrm{H}$ hot spot mutant. Two independent clones of each genotype along with one clone of vector transfected cells were tagged with firefly luciferase and confirmed to express equivalent levels of luciferase (Supplemental Fig. 2A). These cells were injected intravenously into NSG (NOD.Cg-Prkdcscid Il2rgtm1Wil/SzJ) mice, and lung metastases were monitored by bioluminescent imaging (BLI). Mutant p53 conferred increased metastasis in this assay compared with p53-null vector-infected cells (Fig. 2A). Four weeks after tail vein injection, mice injected with tumor cells expressing R72-mut-p53 showed a significant increase in lung metastases compared with the mice injected with tumor cells containing P72-mut-p53 (Fig. 2A). Significant differences in metastatic signal were evident as early as 2 wk after injection (Fig. 2B).
Overall, $85 \%$ of the R72-mut-p53 group (13 out of 15) had detectable lung metastases after $4 \mathrm{wk}$, whereas $60 \%$ of mice in the P72-mut-p53 group (nine out of 15) showed metastases at this time point (data not shown). Histological analysis of lung sections showed that R72mut-p53 tumors revealed an increased total number of nodules (Fig. 2C,D), an increased area of metastases (Fig. 2E), and an overall increased metastatic burden (Fig. 2F) compared with mice injected with tumor cells containing P72-mut-p53. As a control, we assessed proliferation status and p53 levels and again noted no difference in Ki-67 staining in R72-mut-p53 and P72-mut-p53 tumors; we also noted similar levels of mutant p53 in all tumors (Supplemental Fig. 2B-E). We next performed these studies using our PC3 clones following tail vein injection. Again, for the R175H mutation of p53, PC3 cell lines containing R72-mut-p53 showed markedly increased lung metastases (Supplemental Fig. 2F,G), number of lung nodules (Supplemental Fig. 2H,I), and overall area of the lung metastases (Supplemental Fig. 2J) compared with P72mut-p53.
A
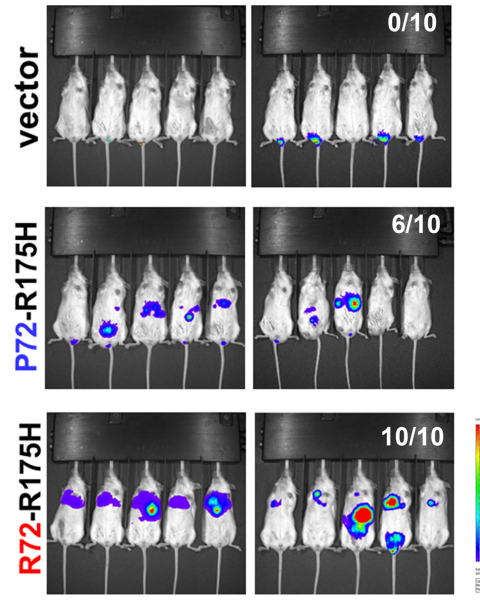

B

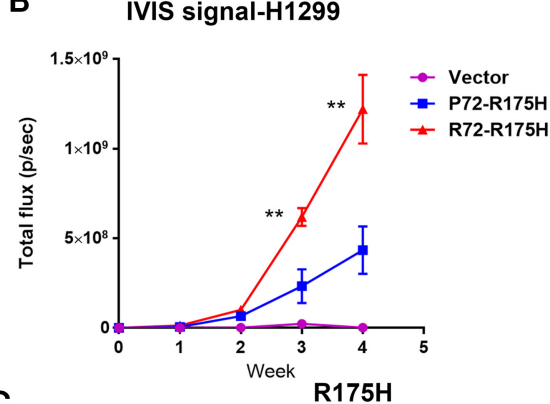

C

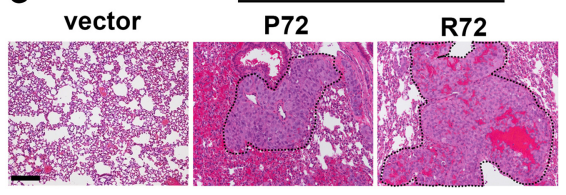

D Number of lung nodules/METs

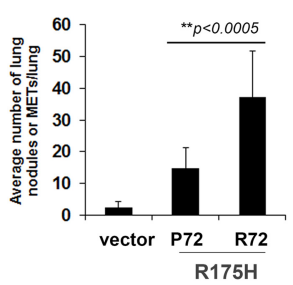

\section{E Area of lung} nodules/METs

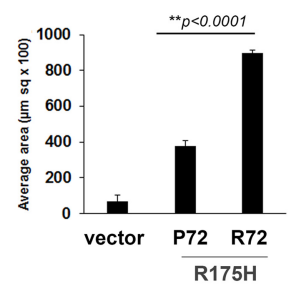

Figure 2. Tumor cells containing R72-mutp53 show increased metastasis in a tail vein assay. (A) Mice injected with H1229 cells expressing either P72-mut-p53 or R72-mut-p53 $(\mathrm{R} 175 \mathrm{H})$ or the vector control were imaged by BLI every week. The luciferase signal for 10 mice in each group is depicted. (B) Graph showing the quantification of the luciferase signal in mice imaged by in vivo imaging system (IVIS) each week for 4 wk. $(* *) P \leq 0.0001 . n=15$ mice for each time point. Error bars represent SD. (C) Hematoxylin and eosin-stained lung sections of mice injected with either the vector control or the $\mathrm{P} 72$ or $\mathrm{R} 72$ variant of the $\mathrm{R} 175 \mathrm{H}$ mutation in p53. Black dotted lines demarcate the lung nodules. Bar, $100 \mu \mathrm{m} .(D, E)$ Quantification of the number $(D)$ and area $(E)$ of lung nodules/metastases. $n=8$ mice per group, selected at random. Error bars represent SEM. ( $F$, left panel) Hematoxylin and eosin-stained lung sections at $4 \times$ magnification showing tumor metastatic burden from the vector control and P72/R72-R175H. The right panel shows quantification of metastatic burden (total area of tumor cells per slide) in each group. $\left(^{*}\right)$ $P<0.05$. Error bars represent SEM. All results in this figure are representative of two independent experiments performed on two independent clones of each genotype.
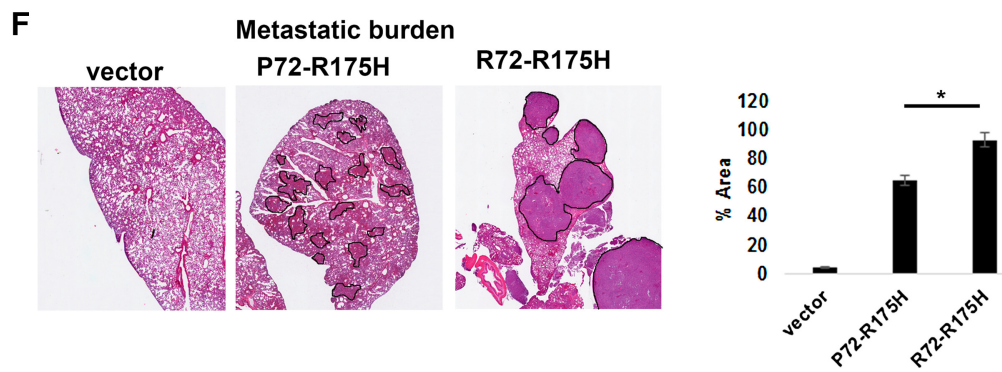
To expand on these findings, we used PC3 cells in bone metastasis assays. Luciferase-tagged PC3 cells expressing P72-R175H or R72-R175H were intracardially injected into experimental mice. Three weeks after intracardiac injection, there was a clear increase in bone metastases of the PC3 prostate carcinoma cells containing R72-R175H (Fig. 3A,B, T=3 wk; data represent two independent clones of each mutant). In addition to the increased overall luciferase signal of the metastases in $\mathrm{R} 72-\mathrm{R} 175 \mathrm{H}$ cells (Fig. 3C), there was also an increased number of bone lesions (Fig. 3D). Histological analyses confirmed the increased bone metastases from R72-R175H cells (Fig. 3E,F).

\section{Mutant p53 associates with and inhibits PGC-1 $\alpha$ to alter cancer cell invasion}

We next sought to delineate the mechanism by which the R72-mut-p53 causes increased cancer cell metastasis. Mutant p53 is known to confer increased invasion by virtue of its ability to inhibit the p53 family members p63 and p73. Both p63 and p73 are known to inhibit cancer cell invasion (Powell et al. 2014), and the codon 72 polymorphism is known to impact binding to p73 (Marin et al. 2000). However, we saw no differences in the RNA levels of the key p63 target genes Cyclin G2 and Sharp-1 or the p73-repressed gene PDGFR $\beta$ (Supplemental Fig. $3 \mathrm{~A}-\mathrm{C})$. We also examined integrin recycling in these cells using phospho-AKT as a downstream readout of integrin signaling (Muller et al. 2009); we saw no differences in pAKT in our R72-mut-p53 and P72-mut-p53 clones (Supplemental Fig. 3D). We therefore chose to address this question in an unbiased manner by performing RNA sequencing (RNA-seq) analysis on our R175H and R273H P72-H1299 and R72-H1299 cells. We found 190 genes to be significantly differentially expressed between R72 and P72 variants in both types of p53 mutant cells. Ingenuity Pathway Analysis (IPA) of this gene set revealed a significant association with the control of oxidative phosphorylation and mitochondrial pathways as well as a significant increase in the expression of genes regulated by PGC-1 $\alpha$ (Fig. 4A; data not shown). This finding prompted us to analyze the transcriptional coactivator PGC-1a.

PGC-1a is a master regulator of oxidative phosphorylation and mitochondrial biogenesis and is known to enhance the ability of some tumor types to undergo distant metastasis (LeBleu et al. 2014). It is also known
A
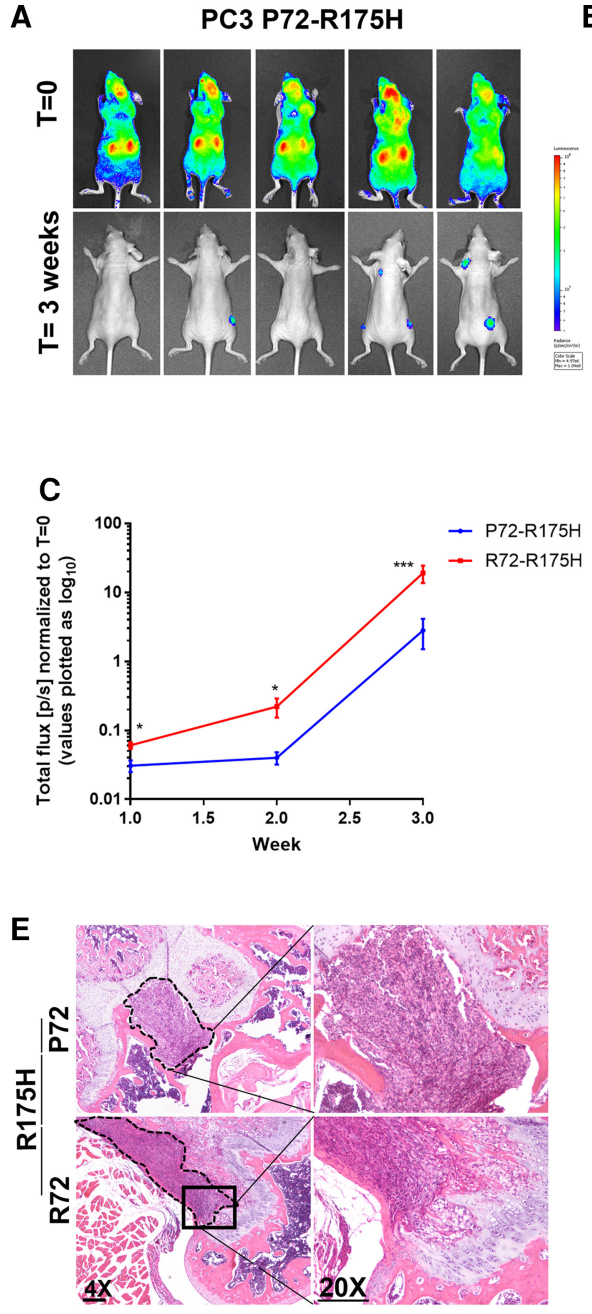

B

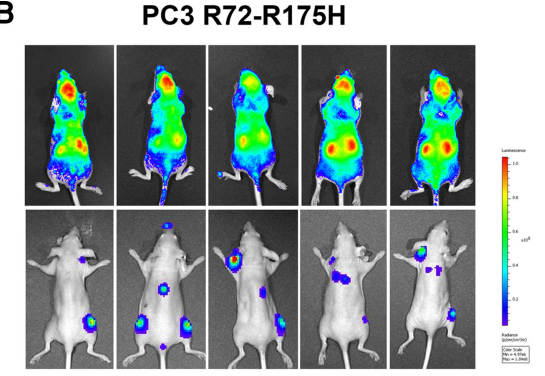

Figure 3. Increased metastasis of $\mathrm{PC} 3$ cells containing R72-mut-p53. (A,B, top panels) Representative IVIS images of mice at $T=0$ wk showing arterial distribution of luciferase-tagged PC3 cells expressing either the P72 or R72 variant of mutant p53-R175H. (Bottom panel) Mice were imaged over time and, at the end of $3 \mathrm{wk}$, showed multiple bone metastases. $n=5$ mice in each group. (C) The total radiance/luciferase signal in mice injected with either the P72 or $\mathrm{R} 72$ variant of $\mathrm{R} 175 \mathrm{H}$, imaged every week for $3 \mathrm{wk}$. Error bars represent SD. $\left({ }^{*}\right) P \leq$ $\left.0.05 ;{ }^{* * *}\right) P \leq 0.0001 . n=5$ mice each genotype. $(D)$ The average number of bone metastases in mice injected with the $\mathrm{P} 72$ or R72 variant of mutant p53-R175H. $n=5$ mice per group. Error bars represent SEM. (E) Representative hematoxylin and eosin-stained longitudinal sections of bone from mice from either the P72-R175H or the R72$\mathrm{R} 175 \mathrm{H}$ group. The left panel shows low (4x) magnification, and the right panel shows high $(20 x)$ magnification. Bar, 100 $\mu \mathrm{m} .(F)$ Quantification of the average area of bone metastases in mice from the P72R175H or R72-R175H group. Error bars represent SEM. $n=5$ mice per group. 
A RNA seq data

\begin{tabular}{lcc} 
Canonical Pathways (FDR<1\%) & N & p value \\
\hline Oxidative Phosphorylation & 18 & $3 \times 10^{-9}$ \\
mTOR Signaling & 24 & $5 \times 10^{-9}$ \\
Mitochondrial Dysfunction & 20 & $2 \times 10^{-7}$ \\
Regulation of elF4 and p70S6K Signaling & 19 & $2 \times 10^{-7}$ \\
EIF2 Signaling & 22 & $6 \times 10^{-7}$ \\
\hline
\end{tabular}

B $\quad$ H1299

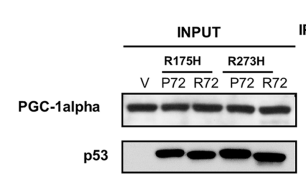

D Proximity ligation assay H1299

(i)

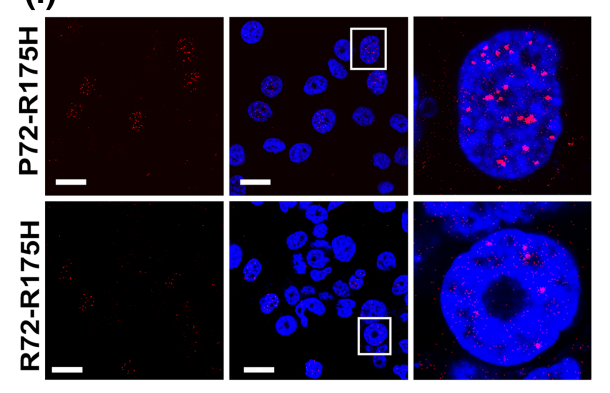

(iii)

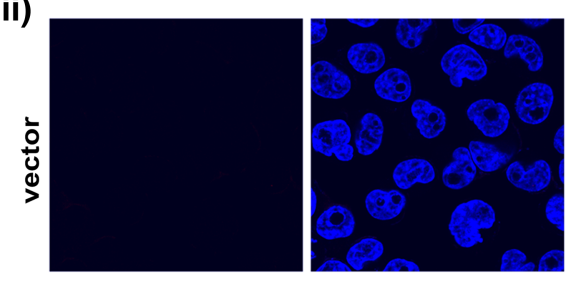

(ii)
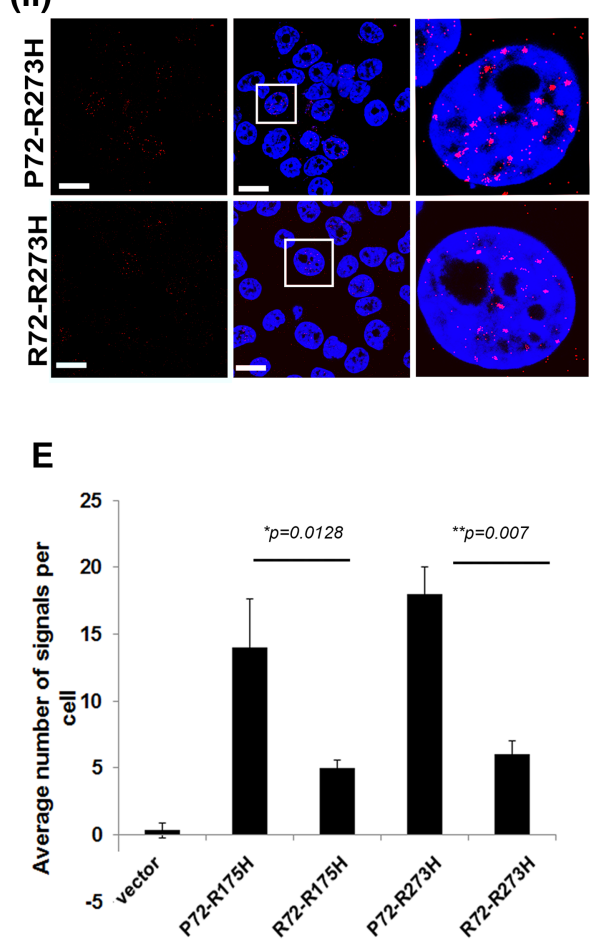

Figure 4. Mutant p53 binds PGC-1 $\alpha$. R72-mut-p53 shows decreased binding compared with P72-mut-p53. (A) Pathway analysis of RNAseq data performed in $\mathrm{H} 1299$ cells containing R72-mut-p53 compared with P72-mut-p53 (R175H and R273H). The top-scoring pathways and their $P$-values are shown. $(B)$ Immunoprecipitation-Western blot analysis of mutant p53 with PGC-1 $\alpha$. Input is shown at the left and the amount of mutant p53 immunoprecipitating with PGC-1 $\alpha$ is shown at the right for the R175H and R273H mutants. (V) Vector control. The data shown are representative of three independent experiments performed in two independent clones for each mutant for each genotype. (C) Immunoprecipitation-Western blot analysis of mutant p53 with PGC-1a in HT-29 (P72-R273H) and SW-620 (R72-R273H). The data depicted are representative of two independent experiments. (D) Proximity ligation assay (PLA) in H1299 cells for PGC-1 $\alpha$ and mutant p53. (Panels i,ii) The left panels show PLA signals for PGC-1 a mutant p53 interaction in cells containing the R175H and R273H mutants, respectively, and the middle panels show cells with the PLA signals for PGC-1a mutant p53 interaction counterstained with DAPI to detect nuclei. Boxed insets in the middle panels are magnified to show representative results from a single cell. Bar, 20 um. (Panel iii) Negative control for PLA in $\mathrm{H} 1299$ cells expressing the vector. The data shown are representative of three independent experiments performed in two independent clones for each mutant for each genotype. (E) Quantification of the average number of PLA signals in H1299 cells expressing the vector control or either P72-mut-p53 or R72-mut-p53. Error bars represent SEM. $n=10$ cells per group, counted from two independent experiments. 
to interact directly with wild-type p53 to the $\mathrm{N}$ terminus of this protein (Sen et al. 2011). To determine whether mutant p53 binds to PGC-1 $\alpha$ and whether the codon 72 variation impacts this binding, we immunoprecipitated PGC1a from H1299 tumor cells and blotted for associated mutant p53. Mutant p53 showed considerable association with PGC-1a; importantly, there was significantly increased binding by P72-mut-p53 in cells containing either the $\mathrm{R} 175 \mathrm{H}$ or $\mathrm{R} 273 \mathrm{H}$ mutations (Fig. 4B). Additionally, there was markedly increased binding of PGC-1 $\alpha$ to mutant p53 in HT-29 cells (P72) compared with SW620 cells (R72) (Fig. 4C). To corroborate these results, we used the proximity ligation assay (PLA), which monitors direct associations between proteins in a quantitative manner. There were equal levels of P72-mut-p53 and R72-mutp53 protein in the nuclei of these tumor cells (Supplemental Fig. 1A), and PLA analyses indicated significantly increased association of the P72-mut-p53 proteins (both $\mathrm{R} 175 \mathrm{H}$ and $\mathrm{R} 273 \mathrm{H}$ ) with PGC-1 $\alpha$ in these cells compared with R72-mut-p53 (Fig. 4D,E).

The combined data suggested that the R72 variant shows decreased association with PGC-1 $\alpha$ along with increased metastasis. PGC-1 $\alpha$ is known to enhance metastasis in certain cancer types (Bhalla et al. 2011; Vazquez et al. 2013; LeBleu et al. 2014). This raised the possibility that mutant p53 might influence the ability of PGC-1 $\alpha$ to interact with and coactivate other transcription factors. Toward this end, we tested three predictions: The first was that cells containing R72-mut-p53 might have an increased level of PGC-1 $\alpha$ target genes compared with P72mut-p53. The second was that tumor cells with R72-mutp53 might have an increased quantity of PGC-1 1 complexes with associated transcription factors, such as PPAR $\gamma$ or HNF4a. The third prediction was that mutant p53 might interfere with the ability of PGC-1 $\alpha$ to function as a coactivator. We assessed the first prediction by analyzing by quantitative RT-PCR (qRT-PCR) the level of six wellknown PGC-1a target genes, again in two independent clones of each genotype in H1299 cells. This analysis revealed slight but consistent and significant increases in the steady-state level of all six PGC-1a target genes in R72-R175H tumor cells compared with P72 (Fig. 5A); furthermore, even PGC-1 $\alpha$, which is itself a PGC-1 $\alpha$ target gene, showed increased expression in R72-mut-p53 cells (Fig. 5B).

We next examined the association between PGC-1 $\alpha$ and PPAR $\gamma$ in tumor cells containing P72-mut-p53 and R72mut-p53. For this, we assessed the quantity of PPAR $\gamma-$ PGC-1a complexes in H1299 cells containing each mutant form of $\mathrm{p} 53$. These PLA results indicate that PGCla binding to PPAR $\gamma$ is enhanced in cells expressing R72-mut-p53 compared with cells expressing P72-mutp53 (Supplemental Fig. 4A,B). We next sought to test the hypothesis that mutant $\mathrm{p} 53$ interferes with PGC-1a function; for this, we relied on the fact that PGC-1a can regulate its own promoter. We transfected a luciferase-tagged PGC-1 $\alpha$ promoter reporter along with PGC-1 $\alpha$ and increasing concentrations of two different mutant forms of p53 (P72-R175H and P72-R273H) in H1299 cells. In this experiment, we found that mutant p53 inhibited the abil- ity of PGC-1 $\alpha$ to coactivate its own promoter in a dose-dependent manner but had no effect on the cotransfected Renilla luciferase control (Supplemental Fig. 4C,D). In these assays, P72-mut-p53 showed a superior ability to inhibit PGC-1 $\alpha$ relative to R72-mut-p53, but it should be noted that R72-mut-p53 still showed an ability to inhibit PGC-1 1 , so this variant is not completely inactive at this function (S Basu, unpubl.). The combined data suggested that PGC-1 $\alpha$ might be required for the ability of mutant p53 to enhance invasiveness. To test this, we silenced PGC-1 $\alpha$ and assessed tumor cell invasion using Boyden chambers. PGC-1 $\alpha$ was effectively silenced using pooled siRNA (Fig. 5B). After silencing, cell invasion was significantly suppressed; notably, this effect was markedly greater in R72-mut-p53 cells, and, again, this silencing equalized the invasive potential of P72-mut-p53 and R72-mut-p53 cells (Fig. 5C,D).

\section{Cells containing R72-mut-p53 show enhanced mitochondrial function and oxidative phosphorylation compared with P72-mut-p53}

To determine whether the increase in PGC-1 a function in R72-mut-p53 cells led to increased markers of mitochondrial biogenesis and function, we next assessed relative mitochondrial content using MitoTracker staining and analyzed the level of mitochondrial RNAs in P72-mutp53 and R72-mut-p53 cells. We also measured oxygen consumption rate and ATP production in two clones of each cell line for the $\mathrm{R} 175 \mathrm{H}$ and $\mathrm{R} 273 \mathrm{H}$ mutants. These analyses revealed that there was markedly increased mitochondrial content in all R72 clones (Fig. 6A). There was also an increased oxygen consumption rate as per Seahorse analysis (Fig. 6B) and increased intracellular ATP levels (Fig. 6C) in all R72-mut-p53 clones. We next examined the mRNA levels of three mitochondrial genes and found that the levels of all three genes (COX1, CYTB, and ND1) were significantly increased in R72-mut-p53 cells compared with P72-mut-p53 (Fig. 6D-F). The combined data support the premise that there is increased PGC-1 $1 \alpha$ activity in R72-mut-p53 tumor cells along with evidence for increased mitochondrial function. To correlate these findings further, we silenced PGC-1 $\alpha$ in P72mut-p53 and R72-mut-p53 cells and found that this equalized the oxygen consumption rate in these clones (Fig. 6G) as well as the ATP level (Fig. 6H).

Increased expression of HNF4 $\alpha$ target genes in R72-mutp53 breast cancer samples

We next sought to determine whether there was increased expression of PGC1 $\alpha$ target genes in tumors from patients with R72-mut-p53. To address this, we analyzed The Cancer Genome Atlas (TCGA) breast cancer database and stratified for samples that contained missense mutations in TP53; these were then assessed for codon 72 polymorphism status using RNA-seq reads for p53 in these samples. We identified 1100 genes whose expression level significantly correlated with the presence of the R72 variant in tumors with mutant p53 $(P<0.05$, Spearman 
A
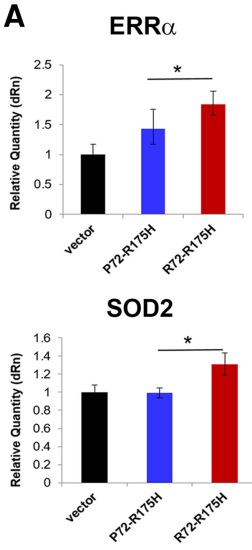

CATALASE

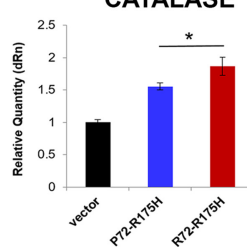

ATP5G1
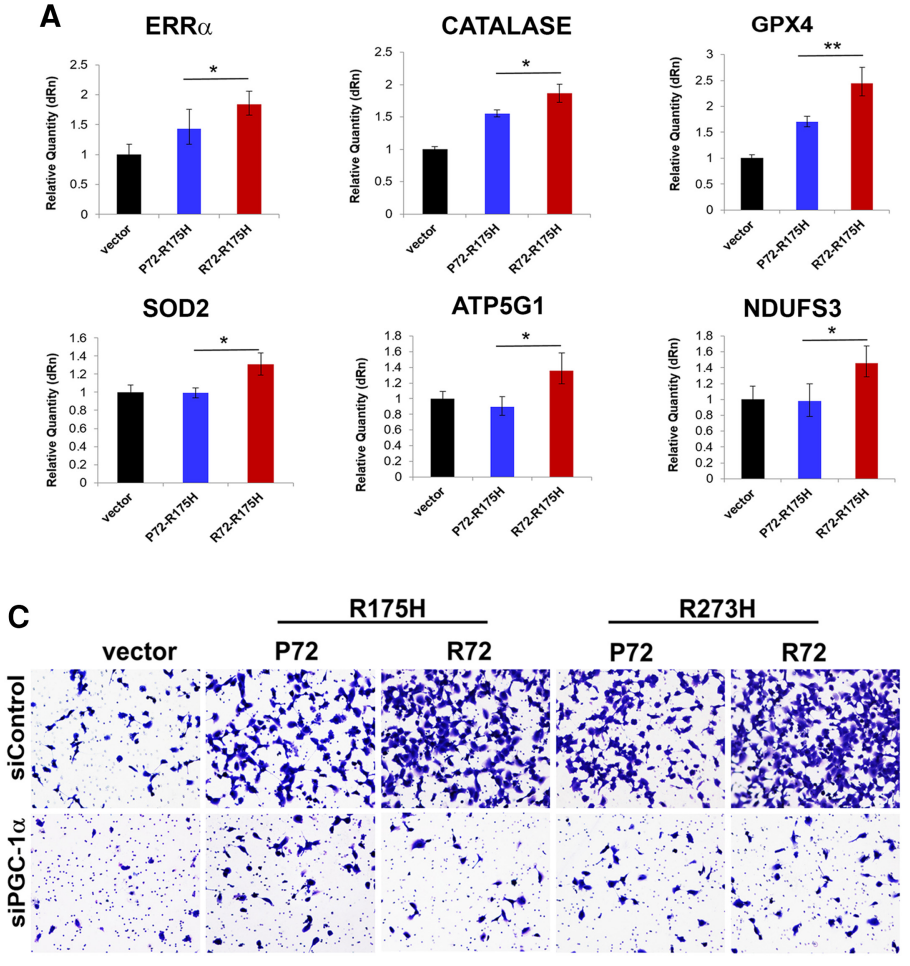

B

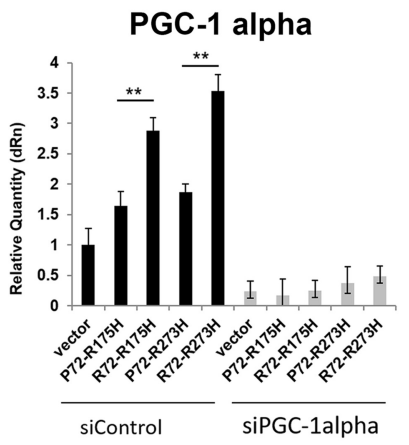

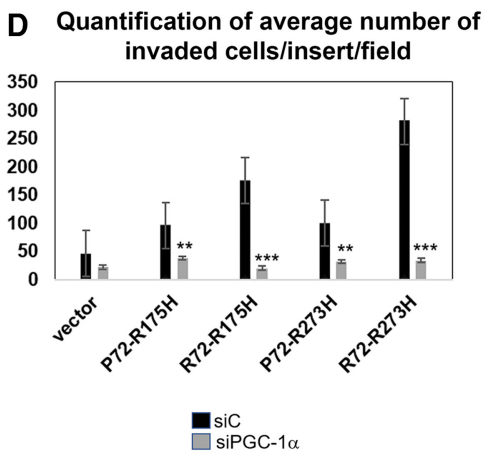

Figure 5. Increased PGC-1 1 target genes in tumors cells with R72-mut-p53; silencing PGC-1 $\alpha$ eliminates the invasiveness differences between P72-mut-p53 and R72-mut-p53. (A) qRT-PCR analysis of mRNA levels of PGC-1a downstream target genes associated with anti-oxidant function (CATALASE, GPX4, and SOD2), transcription (ERRa), and the mitochondria electron transport chain (ATP5g1 and NDUFS3) in H1299 cells containing vector or mutant p53 in cis with P72 or R72. Studies in two replicates were performed in quadruplicate. Error bars mark SD. $\left({ }^{*}\right) P<0.05 ;\left(^{* *}\right) P<0.001$. (B) qRT-PCR of PGC-1 $\alpha$ mRNA levels after siRNA knockdown of PGC-1 $\alpha$ after $48 \mathrm{~h}$ in $\mathrm{H} 1299$ cells, relative to siControl. Error bars represent SEM. Studies are the averaged values of three independent experiments done in duplicate. $(C)$ Representative crystal violet stains of $\mathrm{H} 1299$ cells (vector or P72 or R72 of R175H or R273H) $24 \mathrm{~h}$ following Boyden chamber invasion assays in cells transfected with siControl or siPGC-1 $\alpha$. Invasion assays were performed $48 \mathrm{~h}$ after siRNA treatment. $(D)$ Quantification of the average number of invaded H1299 cells per insert per field following transfection with siControl or siPGC-1a for $48 \mathrm{~h}$. The data depicted are representative of two biological replicates performed in triplicate. $\left(^{* *}\right) P \leq 0.01 ;\left(^{* * *}\right) P \leq 0.0001$. Error bars represent SEM.

correlation). IPA of this gene set revealed a significant enrichment for targets regulated by the transcription factor HNF4 $\alpha(P=0.0008)$ (Fig. 7A); notably, HNF4 $\alpha$ is a key transcription partner for PGCla (Rhee et al. 2006). Altogether, our findings suggested that HNF4 $\alpha$ might show increased association with PGC-1 1 in tumors containing R72-mutp53. Because HNF4a is expressed highly in breast and colon tumors but is not expressed in H1299 or PC3 cells (https://www.proteinatlas.org; our unpublished results), we addressed this question using the HT-29/SW-620 pair of cell lines, which contain the endogenous $\mathrm{R} 273 \mathrm{H}$ mutation. PLA analyses revealed that there is significantly increased association of PGC-1a with HNF4a in SW-620 cells (R72-mut-p53) compared with HT-29 cells (P72mut-p53) (Fig. 7B,C). In line with this, qPCR analysis indicated that the three top HNF4a/PGC-1 1 target genes identified from the breast cancer samples analyzed in Figure 7A (A1BG, CY561D2, and CPA2) also show significantly increased expression in SW-620 cells (Fig. 7D). Consistent with this, chromatin immunoprecipitation (ChIP) experiments showed significantly increased PGC- $1 \alpha$ at the CYB561D2 promoter (Fig. 7E). Our combined data support the premise that R72-mut-p53 is associated with increased PGC-1 $1 \alpha$ function and gene signature compared with P72-mut-p53.

Poorer survival in breast cancer patients with R72-mutp53 compared with P72

We next addressed the possibility that the increased PGCla signature in breast cancer samples containing R72-mut-p53 might be associated with differences in patient outcome. We analyzed 825 RNA-seq samples from breast cancer patients from the TCGA database (BRCA set) that had survival information and contained wildtype or missense mutations in p53 and stratified these for the codon 72 variants using RNA-seq data. This analysis revealed that breast cancer samples containing R72-mut-p53 
A

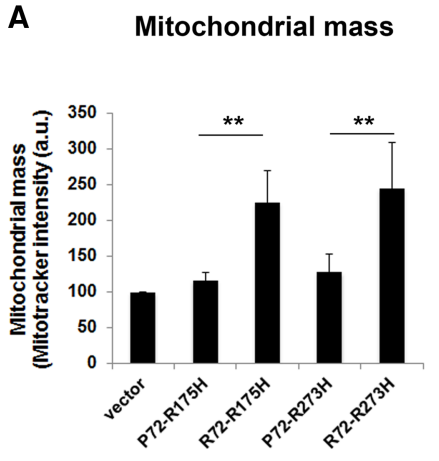

B

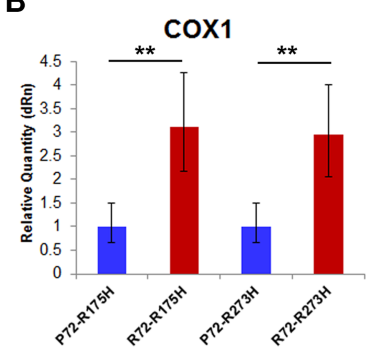

G
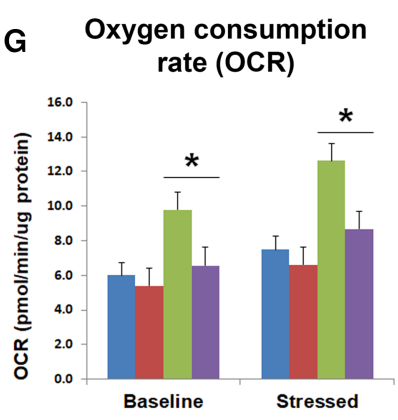

B Oxygen consumption rate (OCR)

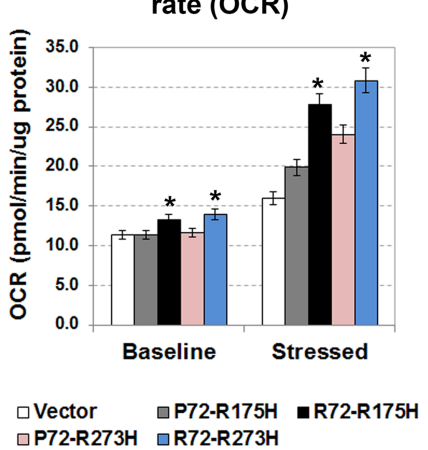

E

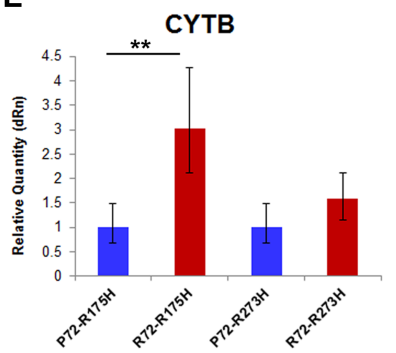

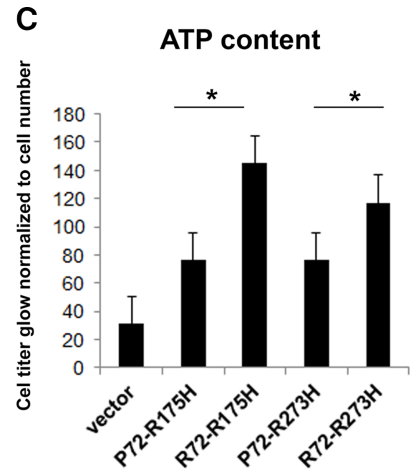

F

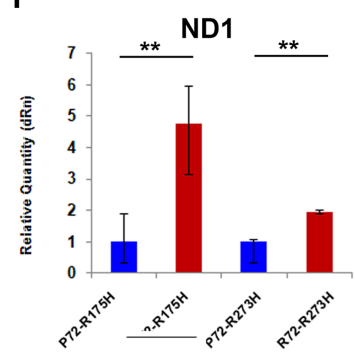

口 P72-R175H sic

- P72-R175H si-PGC1a

$\square$ R72-R175H siC

- R72-R175H si-PGC1a

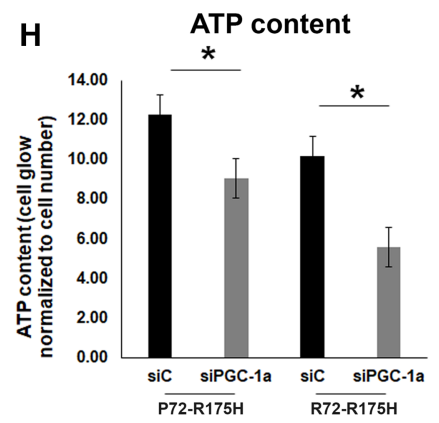

Figure 6. Evidence for increased mitochondrial function in tumor cells containing the R72-mut-p53. (A) Quantification of MitoTracker staining to assess average mitochondrial mass in H1299 cells expressing either the vector control or P72-mut-p53 or R72-mut-p53 (R175H or $\mathrm{R} 273 \mathrm{H})$. Error bars represent SEM. The data depicted are representative of three independent experiments performed in triplicate. $(* *) P$ $<0.001$. (B) Baseline and stressed oxygen consumption rates in H1299 cells expressing either the vector control or P72-mut-p53 or R72mut-p53 (R175H or R273H), as assessed using the Seahorse XFe96 analyzer. Oxygen consumption rate was normalized to the protein content. Error bars represent SD. The data depicted are representative of three independent experiments performed in triplicate. $\left({ }^{*}\right) P<0.05$. (C) ATP content in H1299 cells expressing either the vector control or P72-mut-p53 or R72-mut-p53 (R175H or R273H), as assessed using CellTiterGlo. Error bars represent SEM. The data depicted are representative of three independent experiments performed in triplicate. $\left({ }^{*}\right)$ $P<0.05$. (D-F) qRT-PCR was used to detect mRNA levels of mitochondria-specific genes COX1, CYTB, and ND1 in H1299 cells expressing either the vector control or P72-mut-p53 or R72-mut-p53 (R175H or R273H). Error bars represent SD. The data depicted are representative of three independent experiments performed in triplicate. $\left(^{* *}\right) P<0.001$. $(G)$ Oxygen consumption rate (performed as in $\left.B\right)$ in the indicated cells following silencing of PGC-1 $\alpha$. (H) ATP content (performed as in $C$ ) in the indicated cells following silencing of PGC-1a.

were associated with significantly poorer survival than samples with P72-mut-p53 (hazard ratio of 2.68; $P=$ 0.046). In contrast, there was no association of the codon 72 polymorphism with survival in tumors containing wild-type p53 (Fig. 8A). Overall, an analysis of all 825 individuals indicated that expression of homozygous R72mut-p53 was markedly associated with poorer survival compared with all other samples containing either mutant or wild-type p53 (hazard ratio of 2.07; $P=0.003$ ) (Fig. 8A).

\section{Discussion}

The gain-of-function activities of mutant p53 were clearly elucidated in 1993, when it was shown that mutant p53 isoforms of both human and mouse p53 could endow p53-null tumor cells with an increased ability to form colonies in soft agar and to form tumors in immunocompromised mice (Dittmer et al. 1993). At least one of the gainof-function activities of mutant p53 is impacted by the 


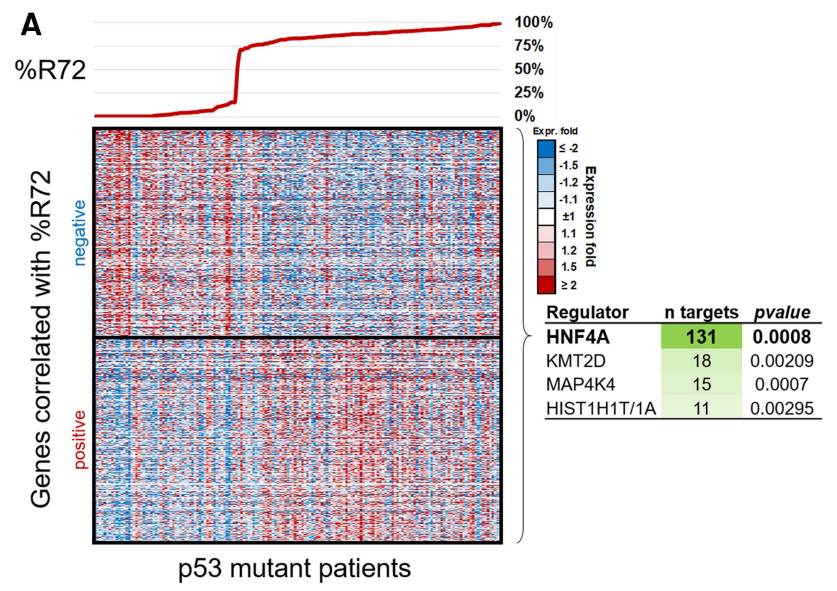

B PGC- $1 \alpha-H N F 4 \alpha$ overlay with Nuclei
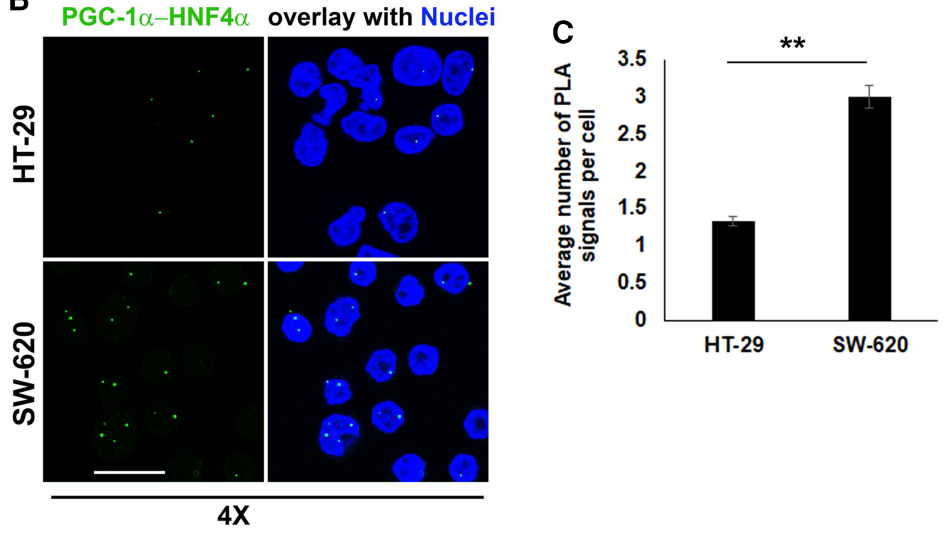

4X
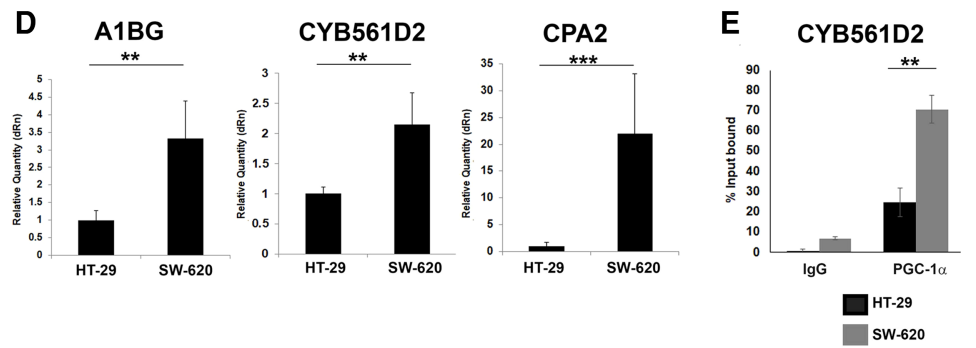

Figure 7. HNF4a signature is enhanced in cells with R72-mut-p53. (A) Expression heat map for genes significantly correlated with the percentage of R72 (as per RNA-seq reads) in patients with p53 missense mutations. $n=161$. Analysis of upstream regulators revealed HNF4a as a top hit with the highest significant number of known targets (131) in the list. $P=0.0008$. $(B)$ PLA in colon cancer cells for PGC-1a and HNF4a. The top and bottom panels show PLA signals for PGC- $1 \alpha$ and HNF4 $\alpha$ interaction in HT-29 cells and SW-620 cells containing the $\mathrm{R} 273 \mathrm{H}$ mutation in p53, respectively. $(C)$ Quantification of the number of PLA signals in HT-29 and SW-620 cells. Error bars show SEM. $\left(^{* *}\right) P<0.001$. (D) qRT-PCR analysis of mRNA levels of the top HNF4a downstream target genes identified from the breast cancer samples from $A(C P A 2, C Y B 516 D 2$, and $A 1 B G)$ in the colon cancer cell lines HT-29 and SW-620 containing mutant p53 (R273H) in cis with P72 and R72, respectively. Studies were performed twice independently in quadruplicate. Error bars mark SEM. $\left.\left(^{* *}\right) P<0.001 ;{ }^{* * *}\right) P<0.0001$. $(E)$ ChIP of HT-29 and SW-620 cells using antisera for IgG or PGC-1a followed by qPCR using primers for CYB516D2. $\left(^{* *}\right) P<0.001$. codon 72 polymorphism. Specifically, this SNP was found to regulate the ability of mutant p53 to bind and inactivate the p53 family member p73; in particular the $\mathrm{R} 72$ variant was more capable of $\mathrm{p} 73$ binding (Marin et al. 2000). However, other "gain-of-function" activities of mutant p53 were not analyzed for an impact of the codon 72 polymorphism, and an overall impact on metastasis and survival has not been done. With regard to metastasis, mutant p53 is known to drive metastasis by inactivation of p63 (Muller et al. 2011). Mutant p53 can also disrupt mammary tissue architecture to a more invasive phenotype by interacting with SREBP1/2 and regulating the mevalonate pathway (Freed-Pastor et al. 2012) or by enhancing integrin recycling coupled with the Rab complex protein (Muller et al. 2009, 2011). In all of these studies, the impact of the codon 72 polymorphism was not investigated.

In this study, we found that PGC-1 $\alpha$, a known positive regulator of metastasis (LeBleu et al. 2014), is bound by mutant forms of $\mathrm{p} 53$. PGC-1 1 interacts with the N-termi- nal domain of $\mathrm{p} 53$, and it is conceivable that amino acid 72 directly influences this protein-protein interaction in a manner similar to MDM2, which also binds to the $\mathrm{N}$ terminus and is influenced in its interaction with p53 by the codon 72 variant (Dumont et al. 2003). We found that the enhanced ability of P72-mut-p53 to bind to PGC-1 $\alpha$ is correlated with decreased mitochondrial content and oxygen consumption in tumor cells expressing this form of mutant p53. We hypothesize that P72-mut-p53 might favor aerobic glycolysis and that this might favor tumor initiation by allowing for increased biomass. Conversely, we found that R72-mut-p53 shows decreased the ability to bind to PGC-1 $\alpha$ and that this is associated with increased mitochondrial function. It is now appreciated that PGC$1 \alpha$ and mitochondria are drivers of metastasis for many tumor types. For example, increased PGC-1 $\alpha$ gives a tumor cell increased bioenergetic flexibility, thereby allowing it to better survive the metabolically demanding metastatic process (Andrzejewski et al. 2017). Another group 

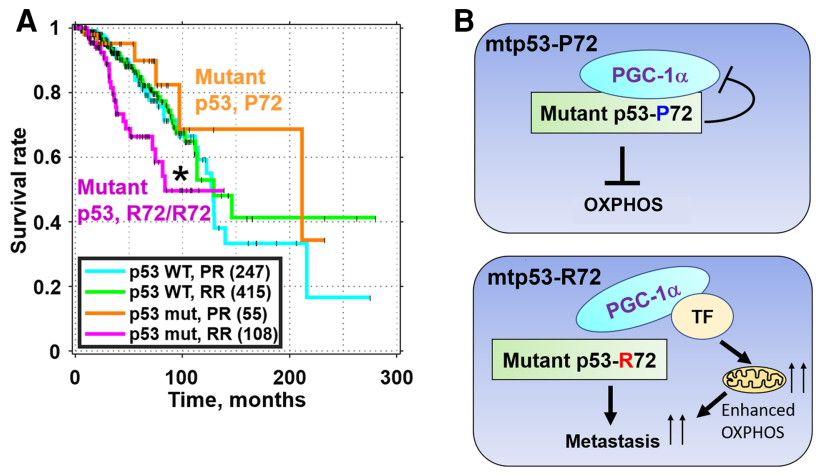

Figure 8. Poorer survival in breast cancer patients with R72mut-p53. (A) Kaplan-Meier survival curves depicting survival data in patients with breast cancer in the presence of both copies of arginine (R72/R72) at codon 72 of mutant p53 (pink line) compared with patients who possess at least one copy of P72 (orange line). Patients with wild-type p53 and one copy of P72 are denoted by the blue line, and patients with wild-type p53 and both copies of R72 are denoted by the green line. (B) Proposed model of how mutant p53 modulates PGC-1 $\alpha$ function to drive cancer cell migration and invasion. P72-mut-p53 is associated with suppressed PGC-1 $\alpha$ activity and reduced oxidative phosphorylation (OXPHOS). Cells containing R72-mut-p53 show increased complex formation of transcription factors (TF) such as HNF4a and PGC- $1 \alpha$ and increased mitochondrial function along with increased invasion and metastasis.

reported that increased mitochondrial function leads to increased superoxide production, causing activation of Src and Pyk2 and thereby promoting tumor cell migration, invasion, and metastasis (Porporato et al. 2014). Recently, one group (Caino et al. 2016) discovered that cortical mitochondria support membrane lamellipodia dynamics, actin cytoskeleton remodeling, and phosphorylation of cell motility kinases, resulting in increased tumor cell motility and invasion. Indeed, inhibition of mitochondrial function by targeting mitochondrial proteostasis can potently inhibit metastatic dissemination of tumor cells (Seo et al. 2016). The combined data suggest that the increased mitochondrial function in R72-mut-p53 tumor cells likely underlies the increased metastasis and poorer survival associated with these tumors. Our results are consistent with two other studies that indicate that high PGC-1a is associated with increased metastasis and poor prognosis in breast cancer (LeBleu et al. 2014; Andrzejewski et al. 2017)

Our combined data best support a model in which the ability of PGC-1 $\alpha$ to bind to transcription factors such as HNF4 $\alpha$ and PPAR $\gamma$ is influenced by mutant p53, and this influence is impacted by the codon 72 variation in mutant p53. We note, however, that a simple model in which mutant p53 binds and inhibits PGC-1 $\alpha$ function is not entirely consistent with our data. For example, we consistently found that tumor cells with mutant p53even P72-mut-p53-show an increased frequency of PGC-1 1 complex formation (such as PPAR $\gamma$ ) (Supplemental Fig. 4A) compared with p53-null cells along with increased expression of PGC-1a target genes (Fig. 5A).
Consistent with this, in HT-29 and SW620 tumor cells, we found that silencing of endogenous mutant p53 causes a marked reduction of PGC-1 a complex formation (S Basu, unpubl.). Therefore, there are clearly multiple levels through which mutant p53 and PGC-1 $\alpha$ can regulate each other. Our data are most consistent with a model in which PGC-1 a complex formation and function are enhanced by mutant p53 in a manner that is significantly impacted by the codon 72 variation (see the model in Fig. 8B).

The p53 family members p63 and p73 play roles in tumor stemness and metastasis. We initially suspected that the differential ability of the codon 72 variants of p53 to enhance metastasis was due to their differential ability to bind and inhibit p63 and/or p73. However, we did not find evidence for increased expression of the p63 target genes Cyclin G2 and Sharp-1 or for decreased expression of the p73-repressed gene PDGF $\beta$ in R72-mutp53 cells - at least not in these cell backgrounds. Additionally, we did not see differences in pAKT levels, which would indicate altered integrin signaling, between P72 and R72 cells (Supplemental Fig. 3). While our data firmly implicate PGC-1 $\alpha$ in the differences in metastasis in P72 and R72 cells, we also found that the lipid metabolism master regulator SREBP1/2 shows a significantly increased ability to bind to R72-mut-p53 (S Basu, unpubl.). Therefore, it remains formally possible that the differential interactions of codon 72 variants with p63, p73, or SREBP1/2 may also play a role in the increased metastasis evident in R72 cells.

One of the interesting features noted many years ago about the codon 72 polymorphism of p53 is that this SNP shows a latitudinal bias with linear increases in the frequency of the R72 allele with increased latitude and colder winter temperatures (Beckman et al. 1994; Shi et al. 2009). This latitudinal bias has never been explained. While some have argued that this bias reflects genetic selection (Beckman et al. 1994), others have refuted this possibility (Sucheston et al. 2011). Genome-wide association studies have found associations between the codon 72 SNP and body mass index (Gloria-Bottini et al. 2011; Bonfigli et al. 2013), waist circumference (Reiling et al. 2012), and predisposition to type II diabetes (Gloria-Bottini et al. 2011); these are all associated with altered metabolism. Indeed, our studies in animal models of diet-induced obesity and cultured cells support the conclusion that this SNP alters the ability of p53 to respond to metabolic stress in the form of either nutrient excess (Kung et al. 2016) or nutrient deprivation (Kung et al. 2017). There is thus a compelling body of evidence in support of the hypothesis that the codon 72 SNP influences the response of p53 to metabolic stress. In light of this premise, it is perhaps not surprising that this SNP also alters the activity of mutant p53 in the regulation of metabolism. Interestingly, a correlation between northern latitudes and breast cancer metastasis has been observed (Grant and Garland 2006). Our study suggests that an impact of the codon 72 polymorphism of p53 may play a role in this observation.

To our knowledge, this is the first time that an impact of the codon 72 polymorphism of mutant p53 on metastasis 
and cancer progression has been assessed. We were unable to find an association between the codon 72 polymorphism of mutant p53 and survival for lung or colorectal cancer (S Basu, unpubl.); therefore, the association between codon 72 of mutant p53 and prognosis may be tumor type-specific. Along these lines, PGC-1 $\alpha$ is negatively associated with metastasis in prostate cancer (Torrano et al. 2016). More information about the roles of PGC-1a and mitochondrial function in the behaviors of different cancers needs to be obtained before we can make broad conclusions about the role of the codon 72 p53 SNP in the prognosis of other cancers. Tumor metabolism is an area of anti-cancer therapies that is actively being pursued. Our data that the codon 72 of p53 influences cancer metabolism suggests that complex I inhibitors such as phenformin might be more effective in tumors with R72-mut-p53 compared with P72-mut-p53; we are actively pursuing this hypothesis with the aim of using this information for personalized medicine approaches.

\section{Materials and methods}

Cell culture, reagents, plasmids, and antibodies

Unless otherwise mentioned, all cell lines were obtained from American Type Culture Collection and used at early passage. Mutant p53 (R175H and R273H) overexpression plasmids encoding proline at codon 72 of p53 were a generous gift from Bert Vogelstein from Johns Hopkins School of Medicine. These plasmids were subjected to site-directed mutagenesis to generate R72 variants (Quick Change II, Stratagene), and all plasmids were verified by sequencing. Cells were transfected with the constructs of interest (5 $\mu \mathrm{g}$ of DNA) with Fugene and selected in $800 \mu \mathrm{g} / \mathrm{mL}$ neomycin for $14 \mathrm{~d}$ to make stable isogenic cell lines. For the luciferase plasmids, H1299 and PC3 cells were labeled with luciferase by lentiviral infection by using the plasmids pLenti-CMV-Puro-Luciferase (Addgene) and pLenti-UbC-RedFLuc-T2A-Puro (Targeting Systems, LP-30). The pLenti-UBc-RedFLuc-T2A-Puro plasmid was a generous gift from Dr. Meenhard Herlyn of The Wistar Institute. The antibodies used were as follows: p53 (DO1) (Calbiochem, OP-43), GAPDH (Cell Signaling Technology, 2118S), TFAM (D5C8) (Cell Signaling Technology, 8076), PGC1a (EMD Millipore, ST1202), PPAR $\gamma$ (R\&D Systems, PPA3409A-00), and Ki67 (D2H10) (Cell Signaling Technology, 9027).

Metastasis assays

Studies were carried out in accordance with the recommendations in the Guide for the Care and Use of Laboratory Animals of the National Institutes of Health $(\mathrm{NIH})$ and approved by the Institutional Animal Care and Use Committee (IACUC) of The Wistar Institute. For tail vein injection studies, 500,000 cells (either H1299 or PC3) expressing mutant p53 (R175H) were tail vein-injected into 6- to 8-wk-old male or female NSG mice. Mice were monitored every week with in vivo imaging system (IVIS) imaging for tumors. For intracardiac injection studies, $5 \times$ $10^{4}$ PC3 cells suspended in $100 \mu \mathrm{L}$ of PBS were injected into the left cardiac ventricle. Mice were injected retro-orbitally with $150 \mathrm{mg} / \mathrm{kg}$ D-luciferin followed by IVIS imaging every week to determine metastatic spread in the bones. At the experimental end point, anesthetized mice $(100 \mathrm{mg} / \mathrm{kg}$ ketamine, $10 \mathrm{mg} / \mathrm{kg}$ xylazine) were imaged with IVIS prior to euthanasia. For all IVIS images, quantification of tumor burden was done with BLI.
BLI was performed using an IVIS Spectrum Xenogen instrument (Caliper Life Sciences) and analyzed using Living Image software.

Immunoblotting, immunoprecipitation-Western, ChIP, and PLAs

For Western blot analysis, $50 \mu$ g of protein was resolved over SDSPAGE gels using precast NuPAGE Bis-Tris gels (Life Technologies) and transferred onto polyvinylidene difluoride membranes (Bio-Rad). Secondary antibodies conjugated to horseradish peroxidase were used at a dilution of 1:10,000 (Jackson Immunochemicals). ECL (Amersham; RPN2232) was then applied to blots, and protein levels were detected using autoradiography. Densitometry quantification of protein signals was performed using ImageJ software (National Institutes of Health). For immunoprecipitation-Western, a total of $1000 \mu \mathrm{g}$ of whole-cell lysate was precleared by protein A-agarose (Cell Signaling Technology, 9863S) or protein G-agarose (Millipore; 16266) beads and incubated with $1 \mu \mathrm{g}$ of antibody overnight at $4^{\circ} \mathrm{C}$. Protein G-agarose beads were then added for $1 \mathrm{~h}$ followed by washes using lysis buffer. Proteins were eluted using $2 \times$ Laemmli sample buffer $(62.5 \mathrm{mmol} / \mathrm{L}$ Tris-HCl at $\mathrm{pH} 6.8,25 \%$ glycerol, $2 \%$ SDS, $0.01 \%$ bromophenol blue) and subjected to Western blot analysis as described earlier. Horseradish peroxidase-conjugated light chain-specific secondary antibody was used (Jackson Immunochemicals). ChIP was performed exactly as described (Kung et al. 2016). To detect interaction or association of proteins by PLA, Duolink in situ starter kits (Sigma, DUO92101, DUO92014) were used, and the manufacturer's protocol was followed exactly as described to perform these studies. PLA signals were quantified using ImageJ software.

\section{Lentiviral transduction of shRNA and siRNA knockdown studies}

Stable cell lines for shRNA knockdowns were generated by infection with the lentiviral vector pLKO.1-puro carrying an shRNA sequence against p53 (CCGGTCAGACCTATGGAAACTACT TCTCGAGAAGTAGTTTCCATAGGTCTGATTTTT) (Sigma, TRCN0000003754). VSVG-pseudotyped lentivirus was generated using 293-FT cells with shRNA constructs and packaging vectors according to the manufacturer's protocols (Invitrogen, K4960-00). Stable cells were selected using 1-5 $\mu \mathrm{g} / \mathrm{mL}$ puromycin, and gene knockdown was confirmed by Western blot analysis. PGC-1a (PPARGC1A) was silenced by transfection with siControl or SMARTpool ON-TARGETplus PPARGC1A/PGC1a siRNA (L-005111-00-0050). Gene knockdown was verified by qRT-PCR.

\section{RNA-seq}

$3^{\prime}$ mRNA-seq libraries were generated from DNase I-treated total RNA using the QuantSeq FWD library preparation kit (Lexogen) according to the manufacturer's directions. Overall library size was determined using the Agilent Tapestation and the DNA 5000 Screentape (Agilent). Libraries were quantitated using realtime PCR (Kapa Biosystems). Libraries were pooled, and high-output single-read 75-base-pair next-generation sequencing was done on a NextSeq 500 (Illumina). RNA-seq data were aligned using Bowtie2 (Langmead and Salzberg 2012) against hg38 version of the human genome, and RSEM version 1.2.12 software (Li and Dewey 2011) was used to estimate raw read counts for each gene using Ensemble v84 transcriptome information. DESeq2 (Love et al. 2014) was used to estimate the significance of the differential expression between sample groups. Overall gene expression changes were considered significant if they passed a false discovery rate (FDR) threshold of $<20 \%$. Gene set enrichment analysis was done using Qiagen's IPA software (Qiagen, http:// 
www.qiagen.com/ingenuity) using the "canonical pathway" option. Pathways with a FDR of $<1 \%$ were considered significantly enriched. The RNA-seq data were deposited to the Gene Expression Omnubus database (http://ncbi.nlm.nih.gov/geo) with accession number GSE109373.

\section{Analysis of TCGA data}

We analyzed RNA-seq data from the TCGA breast cancer (BRCA) database for 838 patients. One-hundred-sixty-seven patients had p53 missense mutations and at least 10 reads spanning the P72R SNP position (rs1042522). The percentage of reads with a G nucleotide (percentage R) was calculated for each patient, the values were correlated with gene expression using Spearman correlation, and genes with a $P$-value of $<0.05$ were considered. Genes were analyzed for enrichment of upstream regulators using Qiagen's IPA software (Qiagen, http://www.qiagen.com/ ingenuity), and protein regulators that had significantly enriched $(P$-value $<0.005)$ targets were considered. For survival analysis, samples from 825 patients with survival information were used. Samples were stratified by wild-type and missense mutated TP53 and by the percentage of R RNA-seq reads, assigning R72/ $\mathrm{R} 72$ status for samples with $>50 \% \mathrm{R}$ variant reads. The significance of survival differences was tested by Cox regression, and results of $P<0.05$ were considered significant.

\section{Statistical analysis of data}

Unless otherwise stated, all experiments were carried out in triplicates and repeated at least in triplicate $(n=3)$ with at least two different clones and pools of cells. For in vitro studies, the unpaired Student $t$-test was performed. For animal experiments, IVIS signal intensities and the number or area of METs were compared using $t$-test between two groups.

\section{Acknowledgments}

We acknowledge the Histotechnology, Laboratory Animal, Bioinformatics, and Imaging Facilities at The Wistar Institute and thank Madeline Good, Joshua Parris, and Anna Budina-Kolomets in the Murphy laboratory for assistance. This work was supported by R01 CA201430 and CA102184 to M.E.M., T32 CA009171 to T.B., and K22 CA181470 to Q.C. Support for the Core Facilities used in this study was provided by Cancer Center Support Grant CA010815 to The Wistar Institute.

Author contributions: S.B., Q.C., and M.E.M. conceived and designed the study. S.B., K.G., T.B., C.-P.K., M.T., J.S., A.W., Q.C., A.V.K., and M.E.M. acquired and analyzed the data. S.B. and M.E.M. wrote the draft of the manuscript. S.B., K.G., T.B., C.-P.K., M.T., J.S., A.W., Q.C., A.V.K., and M.E.M. proofread and revised the manuscript.

\section{References}

Adorno M, Cordenonsi M, Montagner M, Dupont S, Wong C, Hann B, Solari A, Bobisse S, Rondina MB, Guzzardo V, et al. 2009. A Mutant-p53/Smad complex opposes p63 to empower TGFß-induced metastasis. Cell 137: 87-98.

Alexandrova EM, Yallowitz AR, Li D, Xu S, Schulz R, Proia DA, Lozano G, Dobbelstein M, Moll UM. 2015. Improving survival by exploiting tumour dependence on stabilized mutant p53 for treatment. Nature 523: 352-356.

Andrzejewski S, Klimcakova E, Johnson RM, Tabaries S, Annis MG, McGuirk S, Northey JJ, Chenard V, Sriram U, Papadopoli
DJ, et al. 2017. PGC-1 a promotes breast cancer metastasis and confers bioenergetic flexibility against metabolic drugs. Cell Metab 26: 778-787 e775.

Basu S, Murphy ME. 2016. Genetic modifiers of the p53 pathway. Cold Spring Harb Perspect Med 6: a026302.

Beckman G, Birgander R, Sjalander A, Saha N, Holmberg PA, Kivela A, Beckman L. 1994. Is p53 polymorphism maintained by natural selection? Hum Hered 44: 266-270.

Bhalla K, Hwang BJ, Dewi RE, Ou L, Twaddel W, Fang HB, Vafai SB, Vazquez F, Puigserver P, Boros L, et al. 2011. PGCla promotes tumor growth by inducing gene expression programs supporting lipogenesis. Cancer Res 71: 6888-6898.

Bonfigli AR, Sirolla C, Testa R, Cucchi M, Spazzafumo L, Salvioli S, Ceriello A, Olivieri F, Festa R, Procopio AD, et al. 2013. The p53 codon 72 (Arg72Pro) polymorphism is associated with the degree of insulin resistance in type 2 diabetic subjects: a crosssectional study. Acta Diabetol 50: 429-436.

Caino MC, Seo JH, Aguinaldo A, Wait E, Bryant KG, Kossenkov AV, Hayden JE, Vaira V, Morotti A, Ferrero S, et al. 2016. A neuronal network of mitochondrial dynamics regulates metastasis. Nat Commun 7: 13730.

Cooks T, Pateras IS, Tarcic O, Solomon H, Schetter AJ, Wilder S, Lozano G, Pikarsky E, Forshew T, Rosenfeld N, et al. 2013. Mutant p53 prolongs NF- $\mathrm{kB}$ activation and promotes chronic inflammation and inflammation-associated colorectal cancer. Cancer Cell 23: 634-646.

Di Como CJ, Gaiddon C, Prives C. 1999. p73 function is inhibited by tumor-derived p53 mutants in mammalian cells. Mol Cell Biol 19: 1438-1449.

Dittmer D, Pati S, Zambetti G, Chu S, Teresky AK, Moore M, Finlay C, Levine AJ. 1993. Gain of function mutations in p53. Nat Genet 4: 42-46.

Dumont P, Leu JIJ, Della Pietra AC, George DL, Murphy M. 2003. The codon 72 polymorphic variants of $\mathrm{p} 53$ have markedly different apoptotic potential. Nat Genet 33: 357-365.

Freed-Pastor WA, Mizuno H, Zhao X, Langerod A, Moon SH, Rodriguez-Barrueco R, Barsotti A, Chicas A, Li W, Polotskaia A, et al. 2012. Mutant p53 disrupts mammary tissue architecture via the mevalonate pathway. Cell 148: 244-258.

Gaiddon C, Lokshin M, Ahn J, Zhang T, Prives C. 2001. A subset of tumor-derived mutant forms of p53 down-regulate p63 and p73 through a direct interaction with the p53 core domain. Mol Cell Biol 21: 1874-1887.

Gloria-Bottini F, Banci M, Saccucci P, Magrini A, Bottini E. 2011. Is there a role of $\mathrm{p} 53$ codon 72 polymorphism in the susceptibility to type 2 diabetes in overweight subjects? A study in patients with cardiovascular diseases. Diabetes Res Clin Pract 91: e64-e67.

Grant WB, Garland CF. 2006. The association of solar ultraviolet $\mathrm{B}$ (UVB) with reducing risk of cancer: multifactorial ecologic analysis of geographic variation in age-adjusted cancer mortality rates. Anticancer Res 26: 2687-2699.

Kung CP, Leu JI, Basu S, Khaku S, Anokye-Danso F, Liu Q, George DL, Ahima RS, Murphy ME. 2016. The P72R polymorphism of p53 predisposes to obesity and metabolic dysfunction. Cell Rep 14: 2413-2425.

Kung CP, Liu Q, Murphy ME. 2017. The codon 72 polymorphism of p53 influences cell fate following nutrient deprivation. Cancer Biol Ther 18: 484-491.

Lang GA, Iwakuma T, Suh YA, Liu G, Rao VA, Parant JM, Valentin-Vega YA, Terzian T, Caldwell LC, Strong LC, et al. 2004. Gain of function of a p53 hot spot mutation in a mouse model of Li-Fraumeni syndrome. Cell 119: 861-872.

Langmead B, Salzberg SL. 2012. Fast gapped-read alignment with Bowtie 2. Nat Methods 9: 357-359. 
LeBleu VS, O'Connell JT, Gonzalez Herrera KN, Wikman H, Pantel K, Haigis MC, de Carvalho FM, Damascena A, Domingos Chinen LT, Rocha RM, et al. 2014. PGC-1a mediates mitochondrial biogenesis and oxidative phosphorylation in cancer cells to promote metastasis. Nat Cell Biol 16: 992-1003.

Li B, Dewey CN. 2011. RSEM: accurate transcript quantification from RNA-seq data with or without a reference genome. BMC Bioinformatics 12: 323.

Love MI, Huber W, Anders S. 2014. Moderated estimation of fold change and dispersion for RNA-seq data with DESeq2. Genome Biol 15: 550.

Marin MC, Jost CA, Brooks LA, Irwin MS, O'Nions J, Tidy JA, James N, McGregor JM, Harwood CA, Yulug IG, et al. 2000. A common polymorphism acts as an intragenic modifier of mutant p53 behaviour. Nat Genet 25: 47-54.

Matoba S, Kang JG, Patino WD, Wragg A, Boehm M, Gavrilova O, Hurley PJ, Bunz F, Hwang PM. 2006. p53 regulates mitochondrial respiration. Science 312: 1650-1653.

Muller PA, Caswell PT, Doyle B, Iwanicki MP, Tan EH, Karim S, Lukashchuk N, Gillespie DA, Ludwig RL, Gosselin P, et al. 2009. Mutant p53 drives invasion by promoting integrin recycling. Cell 139: 1327-1341.

Muller PA, Vousden KH, Norman JC. 2011. p53 and its mutants in tumor cell migration and invasion. J Cell Biol 192: 209-218.

Muller PA, Trinidad AG, Timpson P, Morton JP, Zanivan S, van den Berghe PV, Nixon C, Karim SA, Caswell PT, Noll JE, et al. 2013. Mutant p53 enhances MET trafficking and signalling to drive cell scattering and invasion. Oncogene 32: 1252-1265.

Olive KP, Tuveson DA, Ruhe ZC, Yin B, Willis NA, Bronson RT, Crowley D, Jacks T. 2004. Mutant p53 gain of function in two mouse models of Li-Fraumeni syndrome. Cell 119: 847-860.

Pfister NT, Fomin V, Regunath K, Zhou JY, Zhou W, Silwal-Pandit L, Freed-Pastor WA, Laptenko O, Neo SP, Bargonetti J, et al. 2015. Mutant p53 cooperates with the SWI/SNF chromatin remodeling complex to regulate VEGFR2 in breast cancer cells. Genes Dev 29: 1298-1315.

Porporato PE, Payen VL, Perez-Escuredo J, De Saedeleer CJ, Danhier P, Copetti T, Dhup S, Tardy M, Vazeille T, Bouzin C, et al. 2014. A mitochondrial switch promotes tumor metastasis. Cell Rep 8: 754-766.

Powell E, Piwnica-Worms D, Piwnica-Worms H. 2014. Contribution of p53 to metastasis. Cancer Discov 4: 405-414.

Reiling E, Lyssenko V, Boer JM, Imholz S, Verschuren WM, Isomaa B, Tuomi T, Groop L, Dolle ME. 2012. Codon 72 polymorphism (rs1042522) of TP53 is associated with changes in diastolic blood pressure over time. Eur I Hum Genet 20: 696-700.

Rhee J, Ge H, Yang W, Fan M, Handschin C, Cooper M, Lin J, Li C, Spiegelman BM. 2006. Partnership of PGC-1 $\alpha$ and HNF4 $\alpha$ in the regulation of lipoprotein metabolism. I Biol Chem 281: 14683-14690.

Sen N, Satija YK, Das S. 2011. PGC-1 $\alpha$, a key modulator of p53, promotes cell survival upon metabolic stress. Mol Cell 44: 621-634.

Seo JH, Rivadeneira DB, Caino MC, Chae YC, Speicher DW, Tang HY, Vaira V, Bosari S, Palleschi A, Rampini P, et al. 2016. The mitochondrial unfoldase-peptidase complex ClpXP controls bioenergetics stress and metastasis. PLoS Biol 14: e1002507.

Shi H, Tan SJ, Zhong H, Hu W, Levine A, Xiao CJ, Peng Y, Qi XB, Shou WH, Ma RL, et al. 2009. Winter temperature and UV are tightly linked to genetic changes in the p53 tumor suppressor pathway in Eastern Asia. Am J Hum Genet 84: 534-541.

Sucheston L, Witonsky DB, Hastings D, Yildiz O, Clark VI, Di Rienzo A, Onel K. 2011. Natural selection and functional genetic variation in the p53 pathway. Hum Mol Genet 20: 1502-1508.

Torrano V, Valcarcel-Jimenez L, Cortazar AR, Liu X, Urosevic J, Castillo-Martin M, Fernandez-Ruiz S, Morciano G, Caro-Maldonado A, Guiu M, et al. 2016. The metabolic co-regulator PGCla suppresses prostate cancer metastasis. Nat Cell Biol 18: 645-656.

Vazquez F, Lim JH, Chim H, Bhalla K, Girnun G, Pierce K, Clish CB, Granter SR, Widlund HR, Spiegelman BM, et al. 2013. PGC1 $\alpha$ expression defines a subset of human melanoma tumors with increased mitochondrial capacity and resistance to oxidative stress. Cancer Cell 23: 287-301.

Wang SP, Wang WL, Chang YL, Wu CT, Chao YC, Kao SH, Yuan A, Lin CW, Yang SC, Chan WK, et al. 2009. p53 controls cancer cell invasion by inducing the MDM2-mediated degradation of Slug. Nat Cell Biol 11: 694-704.

Weissmueller S, Manchado E, Saborowski M, Morris JPt, Wagenblast E, Davis CA, Moon SH, Pfister NT, Tschaharganeh DF, Kitzing T, et al. 2014. Mutant p53 drives pancreatic cancer metastasis through cell-autonomous PDGF receptor $\beta$ signaling. Cell 157: 382-394.

Wolf D, Harris N, Rotter V. 1984. Reconstitution of p53 expression in a nonproducer Ab-MuLV-transformed cell line by transfection of a functional p53 gene. Cell 38: 119-126.

Zhang C, Liu J, Liang Y, Wu R, Zhao Y, Hong X, Lin M, Yu H, Liu $\mathrm{L}$, Levine AJ, et al. 2013. Tumour-associated mutant p53 drives the Warburg effect. Nat Commun 4: 2935.

Zhang C, Liu J, Wu R, Liang Y, Lin M, Liu J, Chan CS, Hu W, Feng Z. 2014. Tumor suppressor p53 negatively regulates glycolysis stimulated by hypoxia through its target RRAD. Oncotarget 5: 5535-5546.

Zhu J, Sammons MA, Donahue G, Dou Z, Vedadi M, Getlik M, Barsyte-Lovejoy D, Al-awar R, Katona BW, Shilatifard A, et al. 2015. Gain-of-function p53 mutants co-opt chromatin pathways to drive cancer growth. Nature 525: 206-211. 


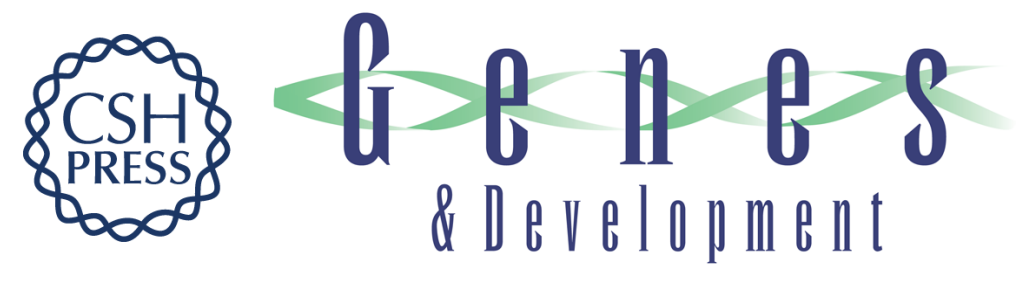

\section{Mutant p53 controls tumor metabolism and metastasis by regulating PGC-1 $\alpha$}

Subhasree Basu, Keerthana Gnanapradeepan, Thibaut Barnoud, et al.

Genes Dev. 2018, 32: originally published online February 20, 2018

Access the most recent version at doi:10.1101/gad.309062.117

\section{Supplemental http://genesdev.cshlp.org/content/suppl/2018/02/19/gad.309062.117.DC1 \\ Material}

Related Content

References

Creative

Commons

License

Email Alerting

Service
SNPing away at mutant p53 activities

Guadalupe J. Ortiz and Guillermina Lozano

Genes Dev. February , 2018 32: 195-196

This article cites 46 articles, 10 of which can be accessed free at:

http://genesdev.cshlp.org/content/32/3-4/230.full.html\#ref-list-1

Articles cited in:

http://genesdev.cshlp.org/content/32/3-4/230.full.html\#related-urls

This article is distributed exclusively by Cold Spring Harbor Laboratory Press for the first six months after the full-issue publication date (see

http://genesdev.cshlp.org/site/misc/terms.xhtml). After six months, it is available under a Creative Commons License (Attribution-NonCommercial 4.0 International), as described at http://creativecommons.org/licenses/by-nc/4.0/.

Receive free email alerts when new articles cite this article - sign up in the box at the top right corner of the article or click here.

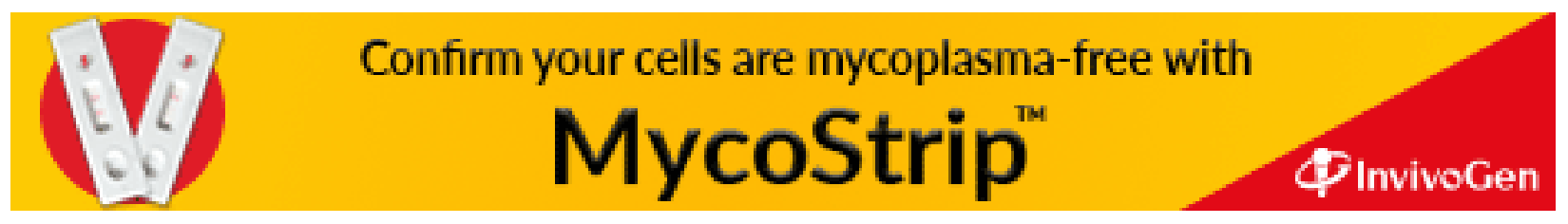

